\title{
Parkinson's disease impacts feedback production during verbal communication
}

\author{
Anahita Basirat ${ }^{1,{ }^{*}}$, Caroline Moreau $^{2,3}$, Dominique Knutsen $^{1}$ \\ ${ }^{1}$ Univ. Lille, CNRS, UMR 9193 - SCALab - Sciences Cognitives et Sciences Affectives, F-59000 Lille, France \\ ${ }^{2}$ Expert center for Parkinson's disease, CHU Lille, Inserm UMR 1171- 2, University of Lille, France \\ ${ }^{3}$ Univ. Lille, Inserm, CHU Lille, U1171-2 - LilNCog - Lille Neuroscience \& Cognition, F-59000 Lille, France \\ ${ }^{*}$ Corresponding author \\ anahita.basirat@univ-lille.fr
}

\begin{abstract}
Background: Parkinson's disease (PD) leads to changes in verbal communications. The focus of most studies to date has been on speech impairment, which is specifically referred to as dysarthria. Although these studies are crucial to understanding the impact of PD on verbal communication, they do not focus on the features of dialogues between people with PD (PwPD) and other people in communicative contexts.

Aims: The current study sought to investigate whether PwPD produce less feedback than typical people during dialogue, thus potentially making it more difficult for them to reach mutual comprehension (i.e., common ground) with their conversational partner.

Methods \& Procedures: A matching task experiment was conducted during which an experimenter described abstract pictures to a participant, who was either a PwPD or a typical participant, so that he or she could organise these pictures in a grid. The participants could produce as much feedback as they liked.

Outcomes \& Results: PwPD were less likely to produce feedback than typical participants. This effect was mainly driven by two specific types of feedback: acknowledgment tokens and hesitations.

Conclusions \& Implications: The results suggest that PD impacts feedback production. This could decrease the communicative abilities of PWPD in interactive contexts by affecting grounding, that is, the ability to build common ground with others. This paper is one of the first to specifically document the production of feedback markers in PwPD. Future studies should examine the extent to which our results, which were obtained in a controlled dialogue task, may be generalized to daily-life conversions. From a clinical perspective, our study points to the necessity of as sessing feedback production, and more generally abilities related to common ground construction and use, during PD progression.
\end{abstract}

Keywords: Parkinson's disease, dialogue, feedback production, matching task, pragmatics 


\section{Introduction}

Parkinson's disease (PD) leads to communication changes in most people with PD (PwPD) (Miller, 2017). These changes, which concern voice, speech and language, deteriorate PwPD's quality of life and impact their socialization. For instance, PwPD tend to adopt the role of the listener during conversational interactions and to exclude themselves from communication (Miller et al., 2006). A large body of studies to date has investigated the acoustics and physiological features of voice and speech in PwPD. Although these studies are crucial to understanding the impact of PD on communication, they do not focus on the features of interactions between PwPD and other people in communicative contexts (Bloch \& Tuomainen, 2017).

To address this issue, a few studies, inspired by methods commonly used with people with aphasia (Milroy \& Perkins, 1992), have analyzed conversational interactions between PwPD and others. The goal of these studies is to understand how PwPD and their conversational partners (e.g. their spouses) manage an interaction. The main focus is usually on potential difficulties of PwPD and their partners during the interaction and on the strategies adopted to "repair" these problems. For instance, Bloch et al. (2015) reported that people with speech disorders, including PwPD, often have difficulties explicitly marking topic transitions. Even when PwPD use appropriate strategies to shift topics during a conversation, e.g., by clearly introducing the new topic at the beginning of the utterance (Yorkston et al., 1996), it is not always sufficient for the conversation to succeed (Saldert \& Bauer, 2017). Other difficulties consist in word retrieval problems (Saldert et al., 2014) and in the decrease of PwPD participation in the conversation due to turn deletion (Griffiths et al., 2012). An important feature of any interaction, which has received less attention in the literature to date, is the production of feedback by PwPD. In the current study, we aim to address this gap by investigating whether and how PD impacts feedback production. In order to do this, we adopt an approach traditionally used in dialogue psychology (e.g., Clark, 1996). We thus hereafter use the term "dialogue" to refer to (goal-oriented) interactions between conversational partners.

A contribution to dialogue is a joint activity which may be divided into two phases: a presentation phase, during which someone produces an utterance, and an acceptance phase, during which his or her conversational partner produces some kind of feedback (verbal or non-verbal) to signal that he or she believes that the utterance 
was understood well enough for current purposes (Clark \& Brennan, 1991; Clark \& Schaefer, 1989). Such feedback enables both partners to add the information produced to their common ground (a representation of the knowledge which both partners are aware of sharing; e.g., Clark, 1996) and to move on to the remainder of the conversation. If the addressee believes that he or she understood correctly the presented utterance, he or she may accept it immediately by saying "yeah", "okay", "I see" or "mhm", by repeating all or part of the utterance, by nodding his or her head or by simply moving on to the following speech turn. In contrast, if the addressee believes that he or she needs additional information to understand the utterance correctly, he or she may initiate a repair phase by signaling his or her hesitation (e.g., by saying "wait"), by asking for additional information, or even by replacing constituents of the utterance (Clark \& Brennan, 1991). In such cases, the information initially presented (and potential modifications made during the repair phase) may only be added to the partners' common ground and the dialogue may only resume after the end of the repair phase.

In their study on the impact of PD on pragmatic communication abilities, McNamara and Durso (2003) mentioned that PwPD provide less feedback to their listeners compared to controls. This observation, as well as others about (for instance) disruptions in conversation initiation and turn taking (McNamara \& Durso, 2003; Hall et al., 2011), are thought to be related to the impact of PD on brain networks involved in the processing of the pragmatic aspects of language (McNamara \& Durso, 2018). But it is noteworthy that no data on feedback production in PD is reported in McNamara and Durso's (2003) study. More recent studies demonstrating pragmatic communication disorders in PD did not analyze feedback production in PwPD either (Hall et al., 2011; Montemurro et al., 2019). However, as described above, feedback plays a central role in the dynamic of the interaction, as difficulties or even the inability to produce feedback makes it difficult for dialogue partners to determine whether or not they have understood each other correctly (e.g., Krauss \& Weinheimer, 1966; Kraut et al., 2003).

In the current study, we used a referential communication task (Clark \& Wilkes-Gibbs, 1986; Bangerter et al., 2020) to examine the impact of PD on feedback production. In this kind of task, one participant (hereafter referred to as the matcher) must place pictures in a grid based on instructions provided by another participant (hereafter referred to as the director). The matcher can provide as much feedback as he or she likes in order to complete the task. The same task is repeated over several trials (the same pictures are used each time, but in a 
different order). The matcher usually provides a lot of feedback during the first trial, as he or she might require more information to understand the director's descriptions of the pictures, to locate the pictures correctly, etc.; the amount of feedback produced then typically decreases over trials (e.g., Knutsen et al., 2019). In the current study, the matcher was a participant (either a PwPD or a control participant) and the director was the experimenter. We examined the amount of feedback produced by the participants. If PD impacts the pragmatic aspects of verbal communication (McNamara \& Durso, 2018), we expect that PwPD will produce less feedback than control participants during the task. To better understand the potential impact of PD on feedback production, we also studied the nature of the feedback produced using an exploratory approach. We examined the likelihood of producing specific feedback markers in each speech turn including acknowledgments, hesitations, replacements, additional information, and repetitions (see Method). If PwPD differ from control participants in producing all of these markers, it would suggest that PD affects feedback production as a whole; in contrast, finding that PD only affects the production of certain feedback markers would shed light on the specific pragmatic functions affected by PD.

\section{Method}

\section{Participants}

Nineteen PwPD (8 females) and sixteen controls (8 females) were included in the experiment. All were French native speakers. The two groups were matched for age (mean \pm SD, PwPD: $66 \pm 9$, controls: $63 \pm 9, t(33)=$ 1.04, $p=0.31)$. In the PD group, the mean duration of disease was 8 years (SD $=7)$. Hearing thresholds were assessed using a screening audiometer at 500,1000 and $2000 \mathrm{~Hz}$ for each ear. The thresholds were then averaged for each participant to obtain an average hearing threshold. Acceptable hearing level was set to less than $40 \mathrm{db} \mathrm{HL}$. Cognitive performance was tested using the Montreal Cognitive Assessment (MOCA) (Nasreddine et al., 2005). The cut-off was set to 22. These two criteria led us to include participants from normal hearing to mild hearing loss and from normal cognition to mild cognitive deficit. The two groups were matched for hearing threshold (mean \pm SD, PwPD: $19 \pm 10$, controls: $18 \pm 9, \mathrm{t}(33)=0.41, p=0.68$ ) and cognitive 
performance (mean \pm SD, PwPD: $27 \pm 3$, controls: $28 \pm 2, \mathrm{t}(29)=-1.31, p=0.20$ ). Note that the MOCA scores for four PwPD were unavailable in the analysis due to data loss. PwPD were under their usual medication (i.e. "on" drug state). The study was conducted in accordance with the tenets of the Helsinki Declaration and approved by the local data protection committee ( $N^{\circ}$ 201810). Before testing, all participants were informed about the study. They signed an informed consent form at the beginning of the study.

\section{Apparatus}

The interaction between the participants and the experimenter were recorded using a double-entry voice recorder (Tascam DR-40).

\section{Materials}

A pool of 24 Tangram figures (see Figure 1 examples) was used in this experiment. These 24 pictures were randomly divided into two sets, hereafter referred to as set $A$ and set $B$.

The experimenter's materials were created by printing 12 pictures (either the 12 pictures from set $A$ or from set B) in a grid on an A4 sheet of paper (see Figure 1). For each set, six such sheets were created. The six sheets featured the same 12 pictures printed in a different (random) order each time. Within each set, the order in which the six sheets were used by the experimenter was randomized, that is, each participant was shown the six sheets in a different (random) order.

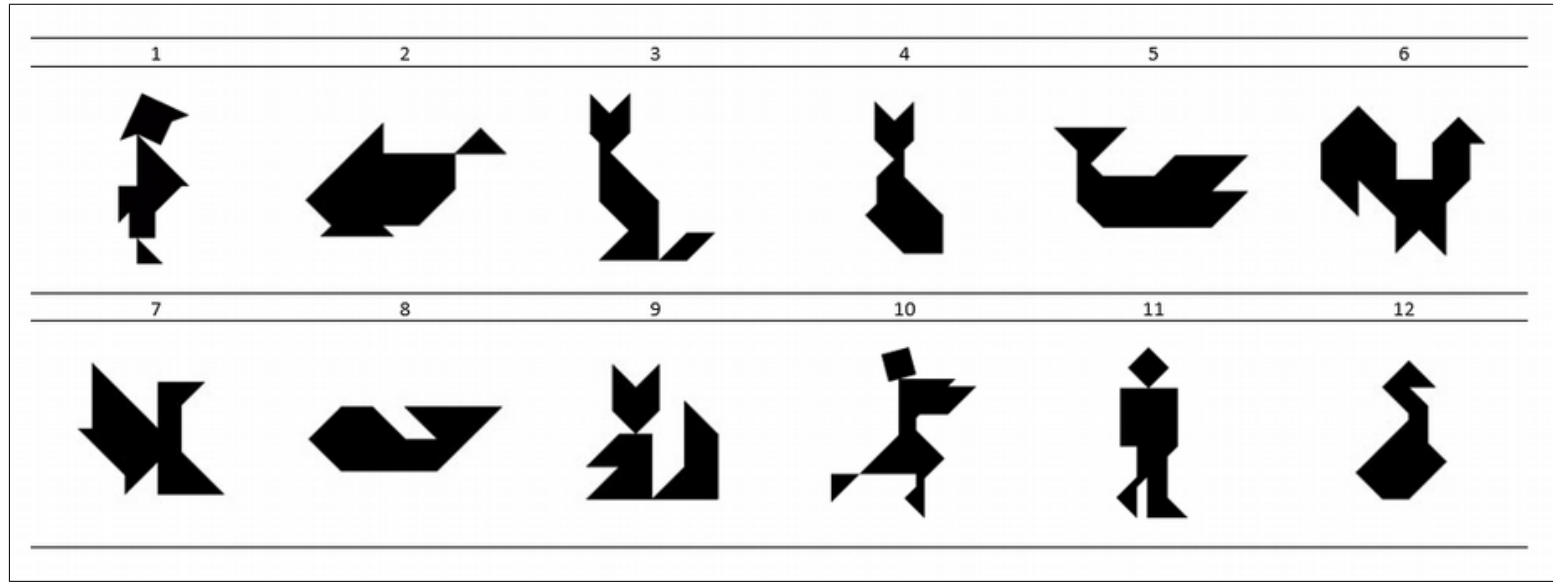


Figure 1. Example of a sheet used by the experimenter.

The participant's materials were created by printing the same 12 pictures (either the 12 pictures from set $A$ or from set $B$ ) on lose paper cards (see Figure 2). These enabled the participant to follow the experimenter's instructions. The participant received the 12 cards corresponding to the set used by the experimenter (e.g., if the experimenter used the set A sheets, the participant received the set A cards).

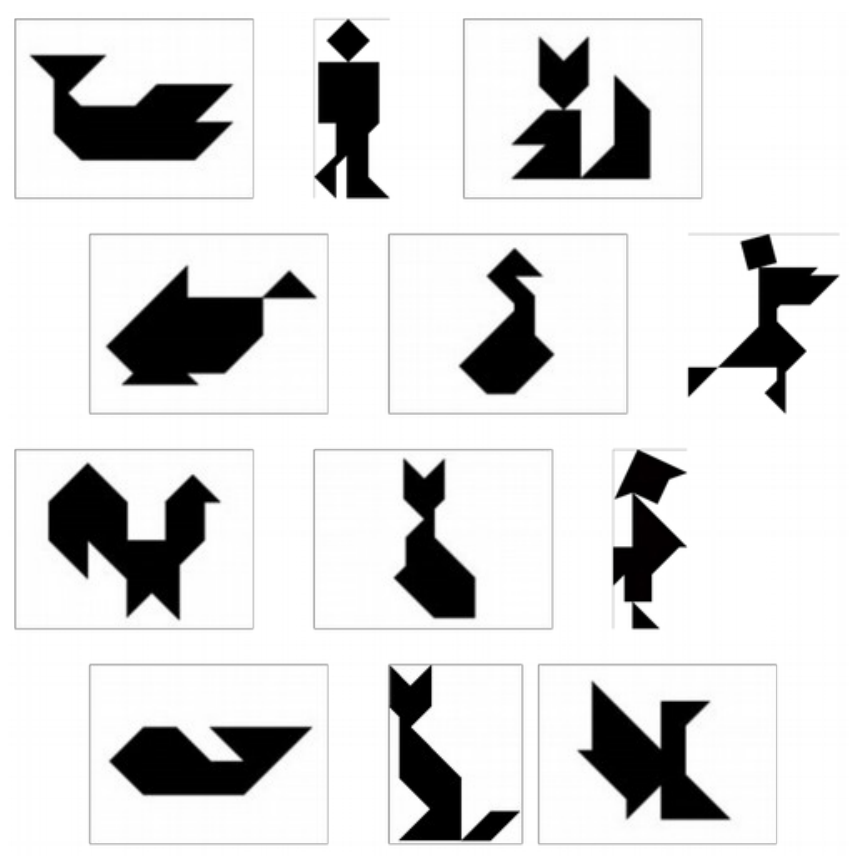

Figure 2. One of the sets used by the participant. Note: This set corresponds to the materials shown in Figure 1.

\section{Task and procedure}

At the beginning of the dialogue task, the participant was informed that he or she was about to perform a task along with the experimenter. Specifically, he or she would be "the matcher" in this task whereas the experimenter would be "the director". He or she was given 12 cards and was told that the experimenter would give him or her instructions to place these cards in the right order. The same task would be repeated several times using the same pictures; the participant would receive feedback regarding the number of pictures placed 
correctly between each trial, but would not be told which mistakes he or she had made (if any). The participant was also told that he or she could go back to the figures placed previously during the same trial. The experimenter then asked the participant if he or she had any questions (and answered the participant's questions if there were any). The experimenter and the participant sat so that they could not see each other's materials or face (ex. opposite to each other, on either side of an opaque partition) in order to prevent nonverbal communication (e.g., looks, head nods, etc.).

Between each trial, the experimenter checked the participant's cards and provided positive or negative feedback, as stated above. The experimenter and the participant did not communicate further between trials, to prevent them from building additional common ground. The experiment was supposed to include six trials. However, in some cases, the participants found the task too difficult. In such cases, the experimenter interrupted the experiment after 20 minutes (she waited until the end of the current trial and announced that the experiment was over without mentioning that the experiment was supposed to include more trials; in such cases, the data from missing trials were not included in the analysis). The task thus lasted approximately 20 minutes for all participants.

A total of six experimenters (all female) took part in running the experiment. All were blind to the authors' hypotheses. The PwPD performed the task at the University Hospital of Lille, France. The control participants performed the same task in a quiet room in various locations (mainly at home). All participants took part in a speech perception task, the results of which are not reported in the current paper, before performing the dialogue task.

\section{Data coding and experimental design}

The dialogues between the participants and the experimenters were transcribed. All words produced were transcribed, including hesitations such as "erm". Non-linguistic signals such as laughs and pauses were not transcribed. The participants' speech was then coded for a number of variables, which are listed below. These variables were the dependent variables (DVs) in the statistical analyses conducted on the data. 
The first DV was the amount of feedback produced by the participant, operationalised as the number of words produced by the participant following the description of each picture by the experimenter.

In order to code the other DVs, we then examined more specifically the content of the speech produced by the participant in response to the description of each picture by the experimenter. We created a coding grid based on work conducted in the context of the collaborative approach to dialogue (see Clark \& Brennan, 1991; Clark \& Wilkes-Gibbs, 1986). The first category of coding considered included all acknowledgments, that is, words and expressions such as "oui" ("yes"), "ouais" ("yeah"), "ok" ("okay"), "je vois" ("I see"), "je comprends" ("I understand"), "c'est bon" ("all good"), etc., which were used by the participant to express the fact that they believed that they had understood the experimenter's instructions ${ }^{1}$.

The second category of coding included all hesitations, that is, words and expressions such as "euh" ("erm"), "attendez" ("wait"), "je ne suis pas sûr-e" ("I'm not sure"), etc. which were used by the participant to express a transient uncertainty. Such uncertainty may be solved by the participant him- or herself in the same speech turn (e.g., "attendez, je ne suis pas sûre, ah si c'est bon" ["wait, I'm not sure, oh yes all good"]) or following new information provided by the experimenter (e.g., P: "attendez, c'est lequel celui-là"; E: "c'est celui avec la bouche ouverte sur le côté"; P: "ah d'accord" [P: "wait, which one is that one"; E: "it's the one with its mouth open on the side"; P: "oh alright"]).

The third category of coding considered included replacements, that is, words and expressions produced by the participant in order to replace the referential expression produced by the experimenter (e.g., E: "et le prochain c'est l'oiseau avec la toute petite aile"; P: "vous voulez dire la baleine pour moi c'est une baleine" [E: "and the next one is the bird with the really small wing"; P: "you mean the whale for me that's a whale"]).

The fourth category of coding considered included additional information, that is, words and expressions produced by the participant in order to add new information to a referential expression produced

${ }^{1}$ This category in fact includes most of Bangerter and Clark's (2003) project markers, which are used by dialogue partners to signal their continued attention and/or their willingness to move on to the remainder of the interaction (see also Knutsen et al., 2018, 2019). 
by the experimenter, but without replacing this referential expression (e.g., E: "je vois une personne"; P: "et c'est comme si portait une assiette" [E: "I see a person"; P: "and it's as if they were carrying a plate"].

The fifth and final category of coding considered included repetitions, that is, situations where the participant repeated at least one of the content words produced by the experimenter (content words were nouns, verbs, adjectives and adverbs; e.g., E: "c'est un oiseau"; P: "euh un oiseau" [E: "it's a bird"; P: "erm a bird"].

These five levels of coding were used to define five binary DVs used in the current experiment: the matcher's response to the director's description either included an acknowledgment or not, a hesitation or not, a replacement or not, a piece of additional information or not, and/or a repetition of the experimenter's speech in the same trial, or not. These five DVs were coded separately, which means that the participants could use several different kinds of markers in their response to the director.

The coding was performed by the research assistants involved in the project and checked by the last author.

The experimental design used in this study included two independent variables (IVs). The first IV, which was between-participants, was the participant's group (PD, control). The second IV, which was withinparticipants, was the trial number (1 through 6; this was a continuous variable).

\section{Results}

The data were analysed using linear (for continuous DVs) or logistic (for binary DVs) mixed models. Mixed models were used because the six experimenters who ran the experiment potentially behaved slightly differently to each other, thus creating "clusters" of participants which might have responded differently to their experimenter. What is more, mixed models may also be used to account for item-related variability (in addition to participant-related variability; see Baayen et al., 2008; Barr et al., 2013). In the current study, the items were the Tangram figures used to perform the task. Finally, mixed models are less sensitive to outliers than most "traditional" statistical analyses. 
The initial random effects structure of the models used in the current study included by-experimenter random intercepts and random slopes corresponding to both IVs, by-participant random intercepts and random slopes corresponding to trial number, and by-item random intercepts and random slopes corresponding to both IVs, in line with Barr et al.'s (2013) recommendation to implement the maximal random effects structure justified by the design. However, doing so sometimes causes convergence issues. When this happened in the current study, we identified the random effects causing these issues (i.e., random effects whose contribution to the model were null) and ran the models for a second time. Importantly, removing random effects which do not contribute significantly to the model does not affect the outcome of the analysis (see Kiernan et al., 2012). The results reported hereafter thus correspond to the converging models; the random effects structure finally used is specified systematically for each analysis.

The basic unit in all analyses was the item, that is, the picture (i.e., for the first DV, we counted the number of words produced by the participant in response to the description of each picture; for the binary DVs, we examined the likelihood of producing each type of feedback in response to the description of each picture).

For some participants, the data from one or several trials was missing (due to the fact that the experimenter interrupted the task after 20 minutes). Thus, the number of observations was not exactly the same across all participants. We accounted for this by applying Satterthwaite's correction (Keselman et al., 1999; Satterthwaite, 1946). When presenting the results of our analyses, for the sake of simplicity, the degrees of freedom are rounded to the nearest integer.

Finally, as mentioned above, each of the analyses reported below included two factors: trial number and group. This implies that the main effect of each IV was calculated in the reference value of the other IV. The reference value of the group IV was the control group, which means that the main effect of trial number was calculated in this group; the group $\mathrm{x}$ trial number interaction would then capture any difference between the control group and the PD group. The reference value of the trial number IV was 1, which means that the main effect of group was calculated at trial 1 . This was done by centring the trial number variable around 1 . The decision to centre this variable around 1 instead of around the mean was based on the fact that the mean is 
3.5, which does not correspond to an actual trial. The group $\mathrm{x}$ trial number interaction would then capture any difference between groups across trials.

All recordings were assessed by a speech and language therapist using the perceptual grid of the French BECD tool (Auzou \& Rolland-Monnoury, 2006). This score includes 5 items assessing vocal quality, phonetic realization, prosody, intelligibility, and natural character of speech. Each item is to be rated from 0 (normal) to 4 (severe impairment). The perceptive score corresponds to the sum of all items and thus varies from 0 (normal speech) to 20 (very severe dysarthria). The speech and language therapist assessed the recordings in a blind manner, i.e., she did not know whether a given recording came from the PD group or the control group. She was also blind to the goal of the study. The mean perceptive scores in PwPD and controls were 2.3 (SD = 2.4, $\min =0, \max =6)$ and $0.6(S D=1.1, \min =0, \max =3)$, respectively. Within the recordings, none of the items was rated 4 (i.e., severe impairment).

\section{Characterisation of the corpus}

The corpus collected in this study included a total of 43,375 words, 34,436 of which were produced by the experimenter playing the role of the director (representing $79.39 \%$ of the corpus) and 8,939 of which were produced by the participant (representing $20.61 \%$ of the corpus).

Participants produced acknowledgment markers in 1,285 cases (54.24\%), hesitation markers in 165 cases (6.96\%), additional information in 151 cases (6.37\%) and repetitions in 397 cases (16.76\%). The number of cases in which a replacement marker was present was very low in comparison to other kinds of feedback markers. We thus decided not to analyse the probability of producing replacement markers as we were worried that such a small number of occurrences would have made the results less reliable.

\section{Analysis \#1: Effect of group and trial number on the number of words produced by the participant}

The data corresponding to this analysis are shown in Figure 3. Large values correspond to the cases where the participants went back to a figure that they had already discussed earlier during the trial, causing them to produce several speech turns and thus a large number of words. 


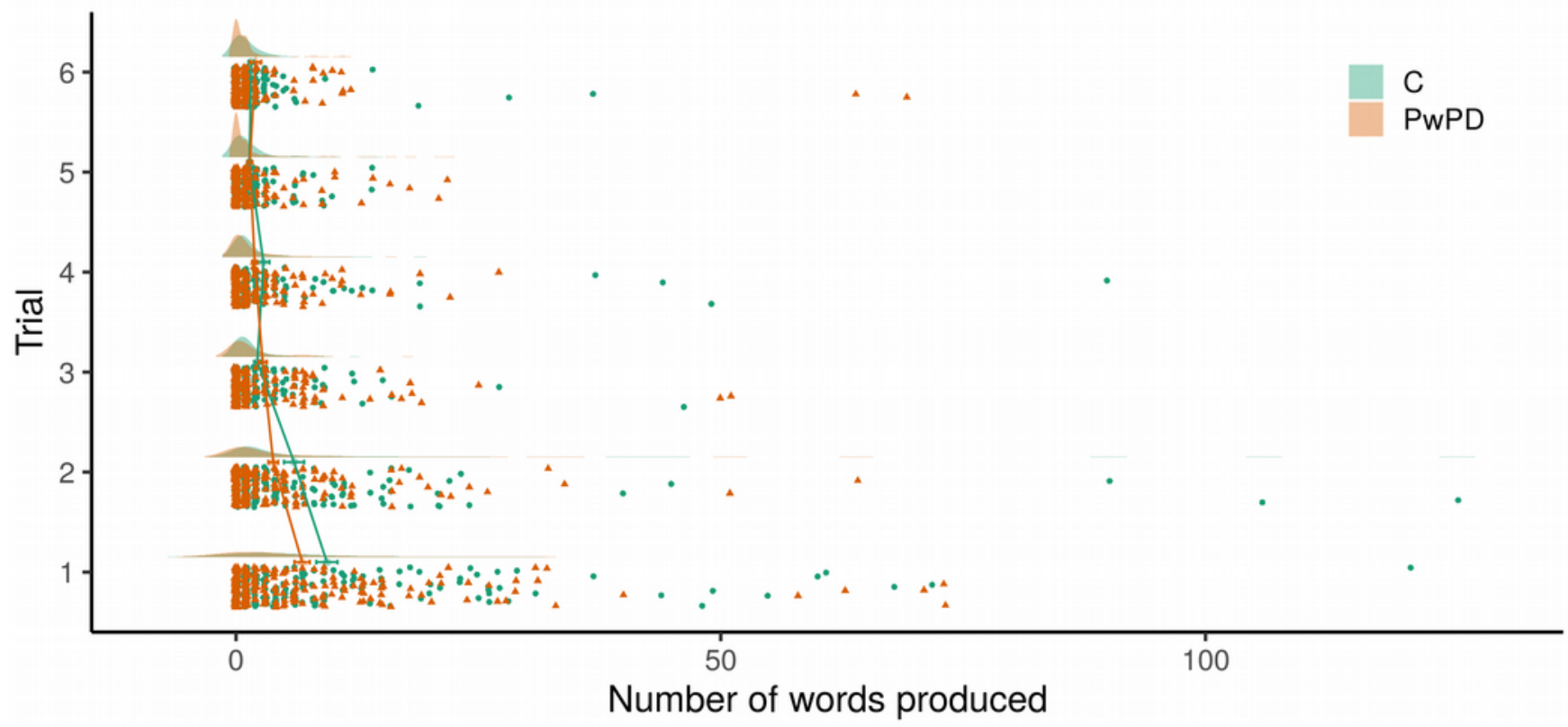

Figure 3. Number of words produced by the participant. For each trial and each group, three plots are shown:

(1) individual data (i.e. number of words produced by each participant) (circles: C, triangles: PwPD), (2) mean and standard error, and (3) a violin plot displaying overall group differences. The colors green and orange represent $C$ and $P D$ groups, respectively.

The results of the analysis are provided in Table 2. As shown in Table 2, no significant effect of group was found. The effect of trial number was significant: an inspection of the associated $b$ coefficient revealed that the number of words produced by the participant decreased over trials. This main effect was qualified by a significant interaction with group, reflecting the fact that the decrease over trials was stronger for participants in the control group than for participants in the PD group. An inspection of Figure 3 suggests that this is due to participants in the control group producing more words in trial 1, thus causing a larger difference between trial 1 and all subsequent trials than in the PD group.

Table 2 
Results of Analysis 1

\begin{tabular}{lll}
\hline Random effect & Estimate & Error \\
\hline By-experimenter random intercepts & 4.65 & 6.87 \\
By-experimenter random slopes corresponding to group & 5.36 & 6.07 \\
By-experimenter random slopes corresponding to trial number & 0.26 & 0.27 \\
By-participant random intercepts & 6.96 & 3.06 \\
By-participant random slopes corresponding to trial number & 0.20 & 0.16 \\
By-item random intercepts & 1.33 & 0.60 \\
Residual & 65.33 & 1.96 \\
\hline
\end{tabular}

\begin{tabular}{lll}
\hline Fixed effect & $b$ & Error \\
\hline Intercept & 7.39 & 1.57 \\
Group: PD & -1.55 & 1.77 \\
Group: Control & 0 & \\
Trial number & -1.50 & 0.28 \\
Trial number x group: PD & 0.56 & 0.26 \\
Trial number x group: Control & 0 & \\
\hline
\end{tabular}

\begin{tabular}{llll}
\hline Effect & Degrees of freedom & $F$ & $p$ \\
\hline Group & 1,5 & 0.77 & .425 \\
Trial number & 1,4 & 24.30 & .010 \\
Trial number x group & 1,22 & 4.61 & .043 \\
\hline
\end{tabular}

Note. The upper panel shows the random effects; the middle panel shows the model parameters; the lower panel shows the $F$ values associated with each effect.

\section{Analysis \#2: Effect of group and trial number on the production of acknowledgments}

The data corresponding to this analysis are shown in Figure 4 (probabilities) and Table 3 (number of pictures for which at least one acknowledgment marker was produced). The results of the analysis are provided in Table 4.

Table 3

Number of Pictures for which each Marker Type was produced

\begin{tabular}{llllllll}
\hline & & Trial 1 & Trial 2 & Trial 3 & Trial 4 & Trial 5 & Trial 6 \\
\hline Acknowledgment markers & Control & 163 & 151 & 111 & 105 & 87 & 87 \\
& PwPD & 150 & 115 & 98 & 87 & 64 & 67 \\
Hesitation markers & Control & 38 & 17 & 7 & 7 & 4 & 6 \\
\multirow{4}{*}{ Additional information } & PwPD & 25 & 25 & 12 & 9 & 8 & 7 \\
\multirow{5}{*}{ Repetition } & Control & 34 & 16 & 11 & 6 & 7 & 4 \\
& PwPD & 37 & 13 & 5 & 7 & 4 & 7 \\
& Control & 48 & 46 & 27 & 36 & 29 & 28 \\
& PwPD & 43 & 38 & 32 & 30 & 21 & 19 \\
\hline
\end{tabular}


As shown in Table 4, a significant effect of group was found. Participants in the control group were more likely to produce acknowledgments than participants in the PD group. The effect of trial number was also significant: an inspection of the associated $b$ coefficient revealed that the probability of producing an acknowledgment decreased over trials. The group $\mathrm{x}$ trial number interaction was non-significant.

C PwPD

\section{Acknowledgment}

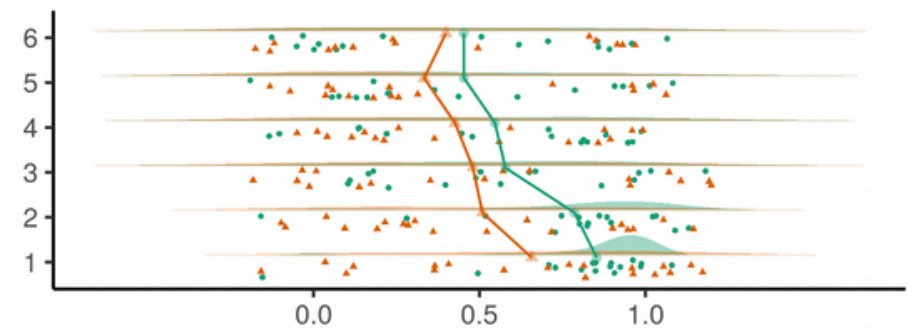

Additional information

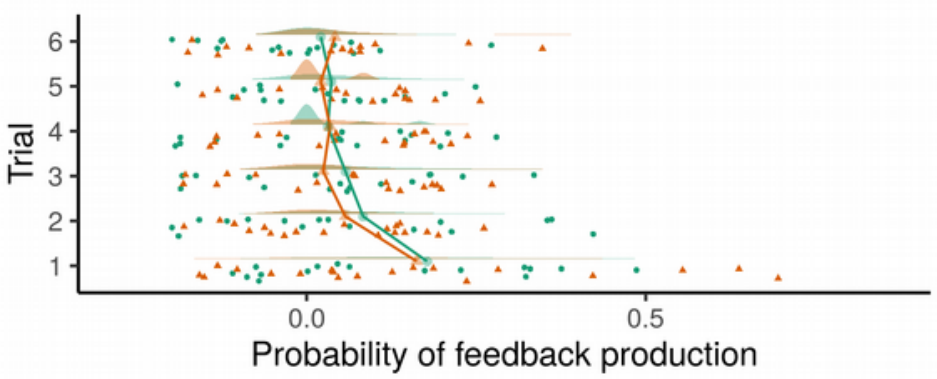

Hesitation

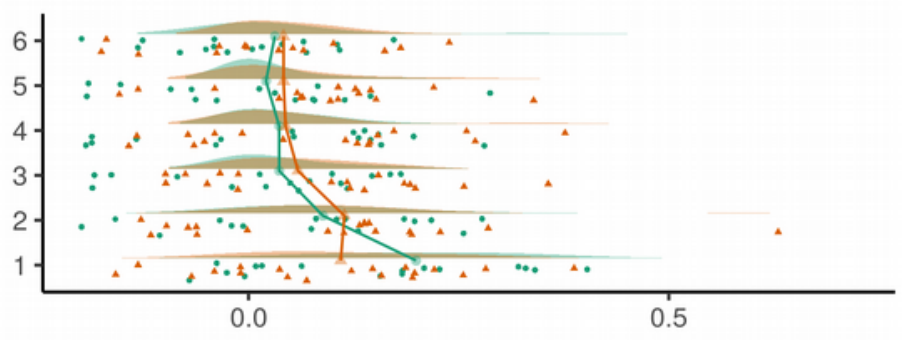

Repetition

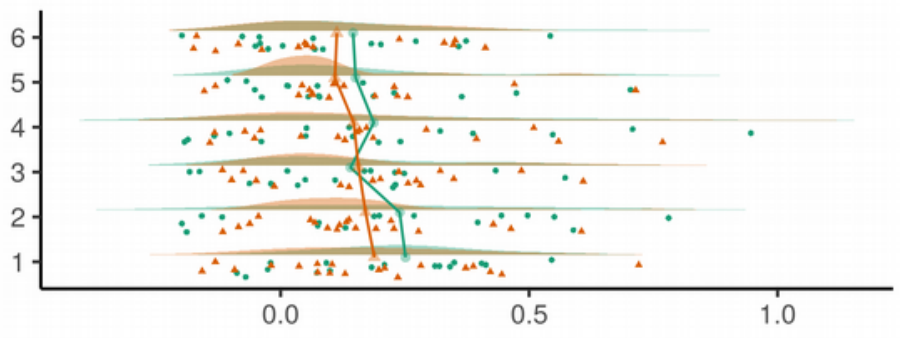

Figure 4: Probability of producing acknowledgments, hesitations, additional information and repetitions. For each feedback marker, three plots are shown per trial and per group: (1) individual data (circles: C, triangles: PWPD), (2) mean, and (3) a violin plot displaying overall group differences. The standard error is not represented here as the DVs are binary: the corresponding dispersion measure (odd ratios) is reported in the results tables. The colors green and orange represent $C$ and $P D$ groups, respectively.

Table 4

Results of Analysis 2

\begin{tabular}{lll}
\hline Random effect & Estimate & Error \\
\hline By-experimenter random intercepts & 1.32 & 1.24 \\
By-participant random intercepts & 2.86 & 0.94
\end{tabular}




\begin{tabular}{|c|c|c|c|}
\hline \multicolumn{4}{|c|}{ By-participant random slopes corresponding to trial number } \\
\hline Fixed effect & $b$ & Error & $\mathrm{OR}(\mathrm{Cl} .95)$ \\
\hline Intercept & 2.08 & 0.68 & \multirow{3}{*}{$0.45(0.10 ; 1.93)$} \\
\hline Group: PD & -1.38 & 0.65 & \\
\hline Group: Control & 0 & & \\
\hline Trial number & -0.62 & 0.13 & \multirow{2}{*}{$\begin{array}{l}\text { - PD group: } 0.68(0.53 ; 0.88) \\
\text { - Control group: } 0.54(0.41 ; 0.70)\end{array}$} \\
\hline & & & \\
\hline Trial number $\mathrm{x}$ group: $\mathrm{PD}$ & 0.24 & 0.18 & \\
\hline Trial number $\mathrm{x}$ group: Control & 0 & & \\
\hline
\end{tabular}

\begin{tabular}{llll}
\hline Effect & Degrees of freedom & $F$ & $p$ \\
\hline Group & 1,28 & 4.46 & .044 \\
Trial number & 1,25 & 32.06 & $<.001$ \\
Trial number x group & 1,25 & 1.82 & .190 \\
\hline
\end{tabular}

Note. The upper panel shows the random effects; the middle panel shows the model parameters (including the odd ratios); the lower panel shows the $\mathrm{F}$ values associated with each effect. OR: odd ratios; $\mathrm{Cl}$ : confidence intervals.

\section{Analysis \#3: Effect of group and trial number on the production of hesitations}

The data corresponding to this analysis are shown in Figure 4 (probabilities) and Table 3 . The results of the analysis are provided in Table 5.

As shown in Table 5, there was no significant effect of group. The effect of trial number was significant: an inspection of the associated $b$ coefficient revealed that the probability of producing a hesitation decreased over trials. This main effect was qualified by a significant interaction with group, reflecting that the decrease over trials was stronger for participants in the control group than for participants in the PD group. An inspection of Figure 4 suggests that this is due to participants in the control group producing more hesitations in Trial, thus causing a larger difference between trial 1 and all subsequent trials than in the PD group.

Table 5

Results of Analysis 3

\begin{tabular}{lll}
\hline Random effect & Estimate & Error \\
\hline By-experimenter random intercepts & 0.29 & 0.29
\end{tabular}




\begin{tabular}{lll} 
By-experimenter random slopes corresponding to group & 0.05 & 0.13 \\
By-experimenter random slopes corresponding to trial number & 0.03 & 0.03 \\
By-participant random intercepts & 0.06 & 0.13 \\
By-participant random slopes corresponding to trial number & 0.04 & 0.03 \\
By-item random intercepts & 0.28 & 0.16 \\
By-item random slopes corresponding to group & 0.03 & 0.10 \\
By-item random slopes corresponding to trial number & 0.02 & 0.02 \\
\hline
\end{tabular}

\begin{tabular}{llll}
\hline Fixed effect & $b$ & Error & $O R(C l .95)$ \\
\hline Intercept & -1.65 & 0.33 & \\
Group: PD & -0.57 & 0.30 & $1.47(0.68 ; 3.17)$ \\
Group: Control & 0 & & \\
Trial number & -0.73 & & - PD group: $0.72(0.55 ; 0.94)$ \\
& & & - Control group: $0.48(0.36 ; 0.65)$ \\
Trial number x group: PD & 0.40 & & \\
Trial number x group: Control & 0 & & \\
\hline
\end{tabular}

\begin{tabular}{llll}
\hline Effect & Degrees of freedom & $F$ & $p$ \\
\hline Group & 1,7 & 3.50 & .104 \\
Trial number & 1,7 & 23.58 & .002 \\
Trial number $x$ group & 1,32 & 7.51 & .010 \\
\hline
\end{tabular}

Note. The upper panel shows the random effects; the middle panel shows the model parameters (including the odd ratios); the lower panel shows the $\mathrm{F}$ values associated with each effect. OR: odd ratios; $\mathrm{Cl}$ : confidence intervals.

\section{Analysis \#4: Effect of group and trial number on the production of additional information}

The data corresponding to this analysis are shown in Figure 4 (probabilities) and Table 3 . The results of the analysis are provided in Table 6.

As shown in Table 6, there was no significant effect of group. The effect of trial number was significant: an inspection of the associated $b$ coefficient revealed that the probability of producing additional information decreased over trials. No significant group $\mathrm{x}$ trial number interaction was found.

Table 6

Results of Analysis 4

\begin{tabular}{lll}
\hline Random effect & Estimate & Error \\
\hline By-experimenter random intercepts & 0.26 & 0.44
\end{tabular}




\begin{tabular}{lll} 
By-experimenter random slopes corresponding to group & 0.25 & 0.40 \\
By-participant random intercepts & 0.56 & 0.33 \\
By-participant random slopes corresponding to trial number & 0.09 & 0.05 \\
By-item random intercepts & 0.24 & 0.19 \\
By-item random slopes corresponding to group & 0.18 & 0.17 \\
By-item random slopes corresponding to trial number & 0.01 & 0.02 \\
\hline
\end{tabular}

\begin{tabular}{llll}
\hline Fixed effect & $b$ & Error & $O R(C l .95)$ \\
\hline Intercept & -1.88 & 0.43 & \\
Group: PD & -0.31 & 0.50 & $0.77(0.22 ; 2.76)$ \\
Group: Control & 0 & & \\
Trial number & -0.60 & 0.12 & - PD group: $0.56(0.43 ; 0.73)$ \\
& & & - Control group: $0.55(0.43 ; 0.71)$ \\
Trial number x group: PD & 0.02 & 0.18 & \\
Trial number x group: Control & 0 & & \\
\hline
\end{tabular}

\begin{tabular}{llll}
\hline Effect & Degrees of freedom & $F$ & $p$ \\
\hline Group & 1,6 & 0.38 & .559 \\
Trial number & 1,25 & 41.62 & $<.001$ \\
Trial number x group & 1,29 & 0.01 & 0.904 \\
\hline
\end{tabular}

Note. The upper panel shows the random effects; the middle panel shows the model parameters (including the odd ratios); the lower panel shows the $\mathrm{F}$ values associated with each effect. OR: odd ratios; $\mathrm{Cl}$ : confidence intervals.

\section{Analysis \#5: Effect of group and trial number on the production of repetitions}

The data corresponding to this analysis are shown in Figure 4 (probabilities) and Table 3 . The results of the analysis are provided in Table 7.

As shown in Table 7, there was no significant effect of group. The effect of trial number was significant: an inspection of the associated $b$ coefficient revealed that the probability of producing a repetition decreased over trials. No significant group $x$ trial number interaction was found.

Table 7

Results of Analysis 5

\begin{tabular}{lll}
\hline Random effect & Estimate & Error \\
\hline By-experimenter random slopes corresponding to group & 1.39 & 1.02 \\
By-experimenter random slopes corresponding to trial number & 0.01 & 0.03
\end{tabular}


By-participant random intercepts

By-participant random slopes corresponding to trial number

\begin{tabular}{llll}
\hline Fixed effect & $b$ & Error & $\mathrm{OR}\left(\mathrm{Cl}_{.95}\right)$ \\
\hline Intercept & -1.41 & 0.56 & \\
Group: PD & -0.29 & 0.79 & $1.09(0.16 ; 7.29)$ \\
Group: Control & 0 & & \\
Trial number & -0.35 & 0.11 & - PD group: $0.82(0.64 ; 1.06)$ \\
& & & - Control group: $0.70(0.54 ; 0.91)$ \\
Trial number x group: PD & 0.16 & 0.14 & \\
Trial number x group: Control & 0 & & \\
\hline
\end{tabular}

\begin{tabular}{llll}
\hline Effect & Degrees of freedom & $F$ & $p$ \\
\hline Group & 1,7 & 0.14 & .720 \\
Trial number & 1,3 & 10.37 & .045 \\
Trial number x group & 1,26 & 1.30 & .264 \\
\hline
\end{tabular}

Note. The upper panel shows the random effects; the middle panel shows the model parameters (including the odd ratios); the lower panel shows the $\mathrm{F}$ values associated with each effect. OR: odd ratios; $\mathrm{Cl}$ : confidence intervals.

\section{Additional analysis: Effect of group and trial number on the probability of producing several different kinds of feedback markers ${ }^{2}$}

One limitation of the analyses reported above is that different types of feedback markers were analyzed separately, raising the question of whether PwPD were less likely than controls to produce several different kinds of feedback while listening to the description of a tangram figure. Indeed, our results up to this point suggest that PwPD are less likely to produce feedback (at least in some cases); it might also be the case that PwPD favor the production of simpler feedback. An additional (unplanned) analysis was conducted in order to address this possibility. This additional analysis required recoding the data: each picture was coded 1 if at least two types of feedback (e.g., acknowledgment and hesitation) among the five types analyzed in this study ${ }^{3}$ were produced by the participant in response to the experimenter's description, and 0 if only one type of feedback

\footnotetext{
${ }^{2}$ We would like to thank a helpful anonymous reviewer for suggesting this additional analysis.

${ }^{3}$ The replacement data were taken into account for the purpose of this recoding. The analysis was also conducted without the replacement data, and the results were the same as those reported here.
} 
(e.g., repetition) was produced. Cases in which no feedback was produced at all were not included in this analysis; in other words, only cases in which at least one feedback marker was produced were included. This level of coding was used as the binary DV, which corresponded to the probability of the matcher producing different kinds of feedback in response to the director's description. The IVs were the same as those used inthe analyses above (i.e., group and trial number).

The data corresponding to this analysis are shown in Figure 5 (probabilities) and in Table 8 . The results of the analysis are provided in Table 9.

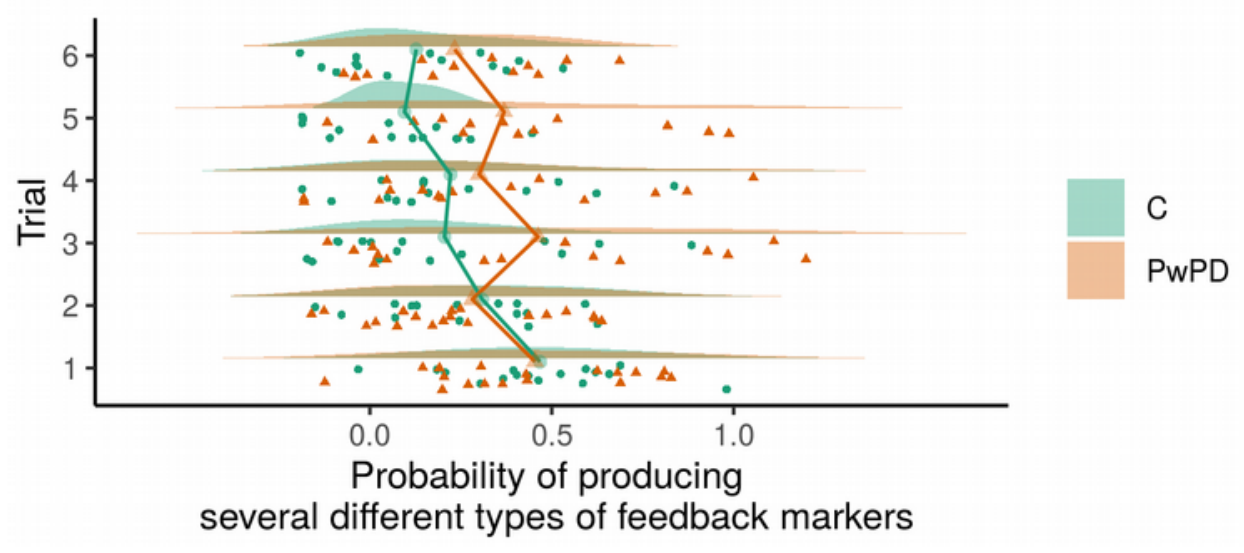

Figure 5: Probability of producing probability of producing several different kinds of feedback markers. For each trial and each group, three plots are shown: (1) individual data (circles: C, triangles: PWPD), (2) mean, and (3) a violin plot displaying overall group differences. The standard error is not represented here as the DVs are binary: the corresponding dispersion measure (odd ratios) is reported in the results tables. The colors green and orange represent $C$ and $P D$ groups, respectively.

Table 8

Number of Pictures for which Different Kinds of Feedback Markers were produced

\begin{tabular}{lll}
\hline & Control & PwPD \\
\hline Trial 1 & 77 & 70 \\
Trial 2 & 50 & 45 \\
Trial 3 & 23 & 36 \\
Trial 4 & 23 & 28 \\
Trial 5 & 13 & 19 \\
Trial 6 & 15 & 15
\end{tabular}


Total $201 \quad 213$

As shown in Table 9, there was no significant effect of group. The effect of trial number was significant: an inspection of the associated $b$ coefficient revealed that the probability of producing multiple kinds of feedback decreased over trials. This main effect was qualified by a significant interaction with group, reflecting that the decrease over trials was stronger for participants in the control group than for participants in the PD group. An inspection of Figure 5 suggests that this is due to participants in both groups were likely to produce different kinds of feedback markers during trial 1; but whereas participants in the control group rapidly switched to producing one type of feedback marker only in the remainder of the trials, PwPD would continue to use different kinds of feedback markers in the remainder of the interaction.

Table 9

Results of the Additional Analysis

\begin{tabular}{lll}
\hline Random effect & Estimate & Error \\
\hline By-experimenter random slopes corresponding to group & 0.03 & 0.20 \\
By-experimenter random slopes corresponding to trial number & 0.01 & 0.03 \\
By-participant random intercepts & 0.68 & 0.28 \\
By-participant random slopes corresponding to trial number & 0.04 & 0.03 \\
By-item random intercepts & 0.03 & 0.08 \\
By-item random slopes corresponding to group & 0.10 & 0.09 \\
By-item random slopes corresponding to trial number & 0.01 & 0.01 \\
\hline
\end{tabular}

\begin{tabular}{llll}
\hline Fixed effect & $b$ & Error & $O R\left(\mathrm{Cl}_{.95}\right)$ \\
\hline Intercept & -0.38 & 0.27 & \\
Group: PD & 0.03 & 0.38 & $1.96(0.80 ; 4.82)$ \\
Group: Control & 0 & & \\
Trial number & -0.51 & 0.10 & - PD group: $0.81(0.65 ; 1.02)$ \\
& & & - Control group: $0.60(0.48 ; 0.76)$ \\
Trial number x group: PD & 0.30 & 0.12 & \\
Trial number x group: Control & 0 & & \\
\hline
\end{tabular}

\begin{tabular}{llll}
\hline Effect & Degrees of freedom & $F$ & $p$ \\
\hline Group & 1,7 & 0.01 & .941 \\
Trial number & 1,4 & 19.77 & .013 \\
Trial number $x$ group & 1,24 & 6.04 & .022 \\
\hline
\end{tabular}


Note. The upper panel shows the random effects; the middle panel shows the model parameters (including the odd ratios); the lower panel shows the $\mathrm{F}$ values associated with each effect. OR: odd ratios; $\mathrm{Cl}$ : confidence intervals.

\section{Discussion}

The purpose of this study was to compare feedback production during dialogue in PWPD versus typical controls. In order to do this, we examined the speech produced by participants playing the role of the matcher in a matching task (e.g., Clark \& Wilkes-Gibbs, 1986).

As in other experiments involving a matching task (e.g., Bangerter et al., 2020), the results revealed that the number of words produced by the participants decreased over trials. Subsequent analyses on specific feedback markers (i.e., acknowledgments, hesitations, additional information, and repetitions) also showed that the likelihood of producing each kind of feedback marker decreased over trials and for both groups. This shows that the acceptance process (e.g., Clark \& Schaefer, 1989; Clark \& Wilkes-Gibbs, 1986), which enables dialogue partners to add information to their common ground, becomes more implicit as the interaction unfolds. When a reference (e.g., "the cat") is produced to designate a tangram figure (Figure 1) for the first time, the matcher must produce some kind of feedback (positive or negative; immediate or delayed) to signal his or her understanding. But once the reference has been added to the partners' common ground during trial 1, such explicit feedback is less necessary and less useful in the remainder of the trials (see also Knutsen et al., 2019).

Crucially, although an overall decrease in feedback production over trials was found in both groups, differences between the two groups of participants were found: PwPD were less likely than control participants to produce acknowledgment markers, and the decrease in the probability of producing hesitations was weaker in the PD group. An inspection of Figure 3 suggests that this difference could be due to PwPD producing fewer words than control participants in the first trials of the task. This is in line with Miller et al.'s report (2006) that PwPD tend to adopt the role of passive listener in an interaction. One possible cause could be the decrease in 
intelligibility and understandability of PwPD due to their speech impairment (see Introduction). The link between speech impairment in PD and verbal interaction has also been highlighted in studies using conversation analysis approach in the home setting (e.g. Griffiths et al., 2015). However, it seems unlikely that our findings are related to speech impairment, since the participants in the current study displayed no severe abnormalities. Rather, this finding is in line with the view that PD impacts brain regions involved in pragmatic aspects of verbal communication (McNamara \& Durso, 2018). This interpretation remains speculative and should be confirmed by studies using a combination of behavioral and neuroimaging methods. In summary, although the origin of the pattern observed in the current study should be examined in future studies, our finding illustrates that PWPD and their dialogue partners may require more time and effort to establish common ground. A more in-depth assessment of speech and language abilities of PwPD, as well as a neuropsychological assessment, would help to better understand the contribution of these different factors to feedback production in PD.

The paradigm used in the current study enabled us to examine how feedback production changes as the dialogue progresses, i.e., while common ground is progressively being co-constructed. The fact that the amount of feedback produced by PwPD decreased as the dialogue unfolds suggests that PwPD remained sensitive to their dialogue partners' needs and used feedbacks to achieve their joint communicative goals. Interestingly, such sensitivity was also reported in studies examining the impact of PD on the production of cospeech gestures (i.e., arm and hand gestures that speakers produced spontaneously for communicative purposes; Humphries et al., 2016; Humphries et al., 2020). These studies show that, although differences between PwPD and controls do exist, the rate of co-speech gestures is similar in PwPD and controls. Future studies should examine whether this degree of sensitivity to partners' needs is sufficient for everyday life conversations to succeed or if the reduced sensitivity of PwPD alters the quality of their interactions with other people.

Our study was the first to specifically study feedback production in PwPD during dialogue. Thus, although we were able to formulate a specific hypothesis regarding the impact of PD on feedback production 
based on the literature (i.e., analysis \#1), our analyses regarding the influence of PD on specific feedback marker production were exploratory (i.e., analyses \#2 to \#5). Interestingly, we observed that the probability of producing acknowledgments was smaller in PwPD than in controls. We also observed that the decrease in the probability of producing hesitations was weaker for PWPD than for controls. No significant effect of group was found for repetitions or additional pieces of information. Although these findings should be investigated further in future research, they already highlight the main difficulties which PwPD are likely to face. PwPD's dialogue partners might find it difficult to determine whether or not they can move on to the remainder of the dialogue, due to PwPD being less likely to produce acknowledgment markers. Dialogue partners might also find it difficult to determine when it is necessary to produce additional information to reach mutual understanding, due to PwPD being less likely to signal their hesitation. To summarise, our findings confirm that PwPD produce less feedback than typical controls during dialogue, in line with our hypothesis of a pragmatic deficit in PwPD (McNamara \& Durso, 2003, Hall et al., 2011; Montemurro et al., 2019). This has important implications for everyday life dialogue, as difficulties or the inability to produce feedback makes dialogue more difficult to manage (e.g., without feedback, dialogue partners must produce longer utterances in order to reach mutual comprehension; e.g., Krauss \& Weinheimer, 1966).

An additional, unexpected finding was that although PwPD were less likely than control participants to produce some of the feedback markers studied, this does not seem to be due to the fact that PwPD were more likely to produce "simpler" feedback (i.e., one type of feedback marker only) than control participants. In fact, the results point in the opposite direction: whereas participants in the control group switched to producing one type of feedback marker only in trials 2-6 (this was often an acknowledgment marker such as "okay" or "mhm"), PwPD would continue to produce different types of feedback markers in these trials. If replicated in future studies on this topic, this finding could imply that PWPD find it difficult to remember which information has already been added to their common ground, leading them to continue to ask questions and to explicitly signal their understanding despite the information already having been marked as shared. 
In the current study, matchers and directors could not see each other during the experiment. The purpose of this was to prevent pairs from communicating non-verbally (e.g., through head nods, gestures, smiles, etc.), but such non-verbal cues can be used in real-life dialogue to signal mutual understanding (see Clark \& Krych, 2004). One possibility is that PwPD use such cues to overcome their difficulty with verbal feedback production in real-life dialogue. The contribution of non-verbal cues to mutual comprehension in PwPD would have to be tested in future work, as the current study was not designed to examine the role of these cues in PWPD. In particular, it would be interesting to examine the production of co-speech gestures in addition to speech production. It has been recently suggested that PD does not impact the use of the co-speech gestures which play a pragmatic role (e.g., gestures used to emphasise a key point to a listener) during narration (Humphries et al., 2020). If this is also the case during dialogue, it could be that PwPD use co-speech gestures to compensate for their reduced ability to produce verbal feedbacks. Such strategies have been reported in people with aphasia and used in some aphasia interventions (for a review, see Clough \& Duff, 2020). Concerning facial expressions, it is noteworthy that PD involves an expressive impairment known as facial masking, a symptom which affects the social life of PwPD (Gunnery et al., 2016; Perepezko et al., 2019). Thus, it is possible that PwPD who suffer from facial masking would find it difficult to use such non-verbal cues as a compensation strategy. This should be addressed in future studies examining the production of both verbal and non-verbal (e.g. co-speech gestures and facial expressions) cues by PwPD in a dialogue setting.

The current work has a number of limitations, one of which being that prosodic cues were not taken into account in our analyses. Yet, these cues play an important role in dialogue settings, for example, to signal appropriate timing for turn-taking (e.g. Edlund \& Heldner, 2005) or to help with syntactic ambiguity in referential contexts (e.g. Snedeker \& Trueswell, 2003). We are thus planning on analysing the prosody of the feedbacks produced during the current study in our future work. Since PD impacts prosody (e.g. Basirat et al., 2018; Skodda \& Schlegel, 2008, Lowit et al., 2018), it would be interesting to examine how prosodic cues contribute to feedback production in PwPD with and without such a deficit. Another limitation concerns the absence of clinical data about the stage of the disease in PwPD who took part in the study. Therefore, we do 
not know whether the ability to produce verbal feedback is affected at an early stage of PD or later on during the progression of the disease. Finally, it should be noted that the sample size was quite small and that the two groups of participants were tested in different locations (i.e., control participants were mainly tested at home whereas PwPD were tested at the hospital).

Our findings are clinically important and suggest that communication deficits in PD go beyond dysarthria, which is the target of behavioral treatments such as the Lee Silverman Voice Treatment (LSVT LOUD), a very effective speech treatment for PD (Ramig et al., 2018). The existence of higher-order language and communication deficits in PD, such as those reported in the current study, demonstrates that an appropriate evaluation of such abilities by speech and language therapists, as well the management of potential deficits, would be beneficial. We believe that the evaluation of PwPD should take into account the theoretical framework used in this study, in particular the role of common ground in communication (Clark, 1996). As indicated above, during communication, interlocutors build and use common ground to ensure mutual comprehension; feedback production plays a key role in this process. Thus, if this ability is affected by PD, PwPD may find it difficult to communicate efficiently with others. In contrast, when this ability is not impaired, or is rehabilitated, verbal interactions would be improved, which may even enable PwPD to compensate for their speech difficulties (i.e. dysarthria), if any. The importance of taking this aspect of communication into account has been recently highlighted in the field of aphasia, leading to a critical review of existing assessment tools by Doedens and Meteyard (2020). We feel that the research and clinical communities working on PD would benefit from such theoretically-driven approaches to assess communicative abilities of PwPD (see also Moreau \& Pinto, 2019). Using appropriate measures to examine how PD impacts communication in interactive contexts will also help to develop more effective therapy programs to improve PwPD's communication skills. The use of collaborative problem-solving paradigms, such as the one used in the current study, with a focus on feedback production, may be an efficient way to work on these skills in a clinical setting.

Future studies should examine the extent to which our results, which were obtained in a controlled dialogue task (i.e., goal-oriented interaction), may be generalized to daily-life conversations. This could be 
achieved by comparing feedback production in a dialogue setting similar to that used in the current study and feedback production in everyday conversation, adopting a conversational analysis approach (e.g. Griffiths et al., 2015; Bloch et al., 2015). We believe that such an effort is worthwhile in order to better understand communication changes caused by $\mathrm{PD}$, to develop relevant assessment tools, and to better manage communicational deficits in PwPD.

\section{Acknowledgments}

We would like to thank all the participants in this study. We are grateful to the experimenters (A.C, A.H., C.A., J.G., M.V., \& N.V.) for their valuable help in collecting data. This work was supported by grants from the Maison Européenne des Sciences de l'Homme et de la Société (MESHS). 


\section{References}

Auzou, P., \& Rolland-Monnoury, V. (2006). BECD: batterie d'évaluation clinique de la dysarthrie. Ortho édition.

Baayen, R. H., Davidson, D. J., \& Bates, D. M. (2008). Mixed-effects modeling with crossed random effects for subjects and items. Journal of memory and language, 59(4), 390-412. https://doi.org/10.1016/j.jml.2007.12.005

Bangerter, A., Mayor, E., \& Knutsen, D. (2020). Lexical entrainment without conceptual pacts? Revisiting the matching task. Journal of Memory and Language, 114, 104-129. https://doi.org/10.1016/j.jml.2020.104129

Barr, D. J., Levy, R., Scheepers, C., \& Tily, H. J. (2013). Random effects structure for confirmatory hypothesis testing: Keep it maximal. Journal of memory and language, 68(3), 255-278. https://doi.org/10.1016/j.jml.2012.11.001

Basirat, A., Patin, C., \& Moreau, C. (2018). Relationship between perception and production of intonation of French in Parkinson's disease. Proceedings of the 9th International Conference onSpeech Prosody 2018, Poznań, Poland, 809-813. https://doi.org/10.21437/SpeechProsody.2018-163

Bloch, S., Saldert, C., \& Ferm, U. (2015). Problematic topic transitions in dysarthric conversation. International Journal of Speech-Language Pathology, 17(4), 373-383. https://doi.org/10.3109/17549507.2014.979879

Bloch, S., \& Tuomainen, J. (2017). Progressive dysarthria and augmentative and alternative communication in conversation: Establishing the reliability of the Dysarthria-in-Interaction Profile. International Journal of Language \& Communication Disorders, 52(1), 3-9. https://doi.org/10.1111/1460-6984.12258

Clark, H. H. (1996). Using language. Cambridge University Press.

Clark, H. H., \& Brennan, S. E. (1991). Grounding in communication. In L. B. Resnick, J. M. Levine, \& S. D. Teasley (Eds.), Perspectives on socially shared cognition (p. 127-149). American Psychological Association.

Clark, H. H., \& Schaefer, E. F. (1989). Contributing to discourse. Cognitive Science, 13, 259-294. https://doi.org/10.1207/s15516709cog1302_7

Clark, H. H., \& Wilkes-Gibbs, D. (1986). Referring as a collaborative process. Cognition, 22, 1-39. https://doi.org/10.1016/0010-0277(86)90010-7 
Clark, H. H., \& Krych, M. A. (2004). Speaking while monitoring addressees for understanding. Journal of Memory and Language, 50, 62-81. https://doi.org/10.1016/j.jml.2003.08.004

Clough, S., \& Duff, M. C. (2020). The role of gesture in communication and cognition: implications for understanding and treating neurogenic communication disorders. Frontiers in Human Neuroscience, 14. https://doi.org/10.3389/fnhum.2020.00323

Doedens, W. J., \& Meteyard, L. (2020). Measures of functional, real-world communication for aphasia: A critical review. Aphasiology, 34(4), 492-514. https://doi.org/10.1080/02687038.2019.1702848

Edlund, J., \& Heldner, M. (2005). Exploring Prosody in Interaction Control. Phonetica, 62(2-4), 215-226. https://doi.org/10.1159/000090099

Griffiths, S., Barnes, R., Britten, N., \& Wilkinson, R. (2012). Potential Causes and Consequences of Overlap in Talk between Speakers with Parkinson's Disease and Their Familiar Conversation Partners. Seminars in Speech and Language, 33(1), 27-43. https://doi.org/10.1055/s-0031-1301161

Griffiths, S., Barnes, R., Britten, N., \& Wilkinson, R. (2015). Multiple repair sequences in everyday conversations involving people with Parkinson's disease. International Journal of Language \& Communication Disorders, 50(6), 814-829. https://doi.org/10.1111/1460-6984.12178

Gunnery, S. D., Habermann, B., Saint-Hilaire, M., Thomas, C. A., \& Tickle-Degnen, L. (2016). The Relationship between the Experience of Hypomimia and Social Wellbeing in People with Parkinson's Disease and their Care Partners. Journal of Parkinson's Disease, 6(3), 625-630. https://doi.org/10.3233/JPD-160782

Hall, D., Ouyang, B., Lonnquist, E., \& Newcombe, J. (2011). Pragmatic Communication is Impaired in Parkinson Disease. International Journal of Neuroscience, 121(5), 254-256. https://doi.org/10.3109/00207454.2010.550389 Humphries, S., Holler, J., Crawford, , T. J. \& Poliakoff, E. (2020). Co-speech gestures are a window into the effects of Parkinson's disease on action representations. Journal of Experimental Psychology: General.

Humphries, S., Holler, J., Crawford, T. J., Herrera, E., \& Poliakoff, E. (2016). A third-person perspective on cospeech action gestures in Parkinson's disease. Cortex, 78, 44-54. https://doi.org/10.1016/i.cortex.2016.02.009 
Keselman, H. J., Algina, J., Kowalchuk, R. K., \& Wolfinger, R. D. (1999). The analysis of repeated measurements: A comparison of mixed-model satterthwaite $\mathrm{F}$ tests and a nonpooled adjusted degrees of freedom multivariate test. Communications in Statistics-Theory and Methods, 28, 2967-2999. https://doi.org/10.1080/03610929908832460

Knutsen, D., Bangerter, A., \& Mayor, E. (2019). Procedural coordination in the matching task. Collabra: Psychology, 5, 3. https://doi.org/10.1525/collabra.188

Krauss, R. M., \& Weinheimer, S. (1966). Concurrent feedback, confirmation, and the encoding of referents in verbal communication. Journal of Personality and Social Psychology, 4, 343-346. https://doi.org/10.1037/h0023705

Kraut, R. E., Fussell, S. R., \& Siegel, J. (2003). Visual information as a conversational resource in collaborative physical tasks. Human-Computer Interaction, 18, 13-49. https://doi.org/10.1207/S15327051HCI1812 2

Lowit, A., Marchetti, A., Corson, S., \& Kuschmann, A. (2018). Rhythmic performance in hypokinetic dysarthria: Relationship between reading, spontaneous speech and diadochokinetic tasks. Journal of Communication Disorders, 72, 26-39. https://doi.org/10.1016/j.jcomdis.2018.02.005

McNamara, P., \& Durso, R. (2003). Pragmatic communication skills in patients with Parkinson's disease. Brain and Language, 84(3), 414-423. https://doi.org/10.1016/S0093-934X(02)00558-8

McNamara, P., \& Durso, R. (2018). The dopamine system, Parkinson's disease and language function. Current Opinion in Behavioral Sciences, 21, 1-5. https://doi.org/10.1016/i.cobeha.2017.10.010

Miller, N. (2017). Communication changes in Parkinson's disease. Practical Neurology, practneurol-2017001635. https://doi.org/10.1136/practneurol-2017-001635

Miller, N., Noble, E., Jones, D., \& Burn, D. (2006). Life with communication changes in Parkinson's disease. Age and Ageing, 35(3), 235-239. https://doi.org/10.1093/ageing/afj053

Milroy, L., \& Perkins, L. (1992). Repair strategies in aphasic discourse; towards a collaborative model. Clinical Linguistics \& Phonetics, 6(1-2), 27-40. https://doi.org/10.3109/02699209208985517

Montemurro, S., Mondini, S., Signorini, M., Marchetto, A., Bambini, V., \& Arcara, G. (2019). Pragmatic Language Disorder in Parkinson's Disease and the Potential Effect of Cognitive Reserve. Frontiers in Psychology, 10. https://doi.org/10.3389/fpsyg.2019.01220 
Moreau, C., \& Pinto, S. (2019). Misconceptions about speech impairment in Parkinson's disease. Movement Disorders, mds.27791. https://doi.org/10.1002/mds.27791

Nasreddine, Z. S., Phillips, N. A., Bédirian, V., Charbonneau, S., Whitehead, V., Collin, I., Cummings, J. L., \& Chertkow, H. (2005). The Montreal Cognitive Assessment, MoCA: A Brief Screening Tool For Mild Cognitive Impairment. Journal of the American Geriatrics Society, 53(4), 695-699. https://doi.org/10.1111/i.1532$\underline{5415.2005 .53221 . x}$

Perepezko, K., Hinkle, J. T., Shepard, M. D., Fischer, N., Broen, M. P. G., Leentjens, A. F. G., Gallo, J., \& Pontone, G. M. (2019). Social Role Functioning in Parkinson's disease: A mixed-methods systematic review. International Journal of Geriatric Psychiatry, 34(8), 1128-1138. https://doi.org/10.1002/gps.5137

Ramig, L., Halpern, A., Spielman, J., Fox, C., \& Freeman, K. (2018). Speech treatment in Parkinson's disease: Randomized controlled trial (RCT). Movement Disorders, 33(11), 1777-1791. https://doi.org/10.1002/mds.27460

Saldert, C., \& Bauer, M. (2017). Multifaceted Communication Problems in Everyday Conversations Involving People with Parkinson's Disease. Brain Sciences, 7(10), 123. https://doi.org/10.3390/brainsci7100123

Saldert, C., Ferm, U., \& Bloch, S. (2014). Semantic trouble sources and their repair in conversations affected by Parkinson's disease. International Journal of Language \& Communication Disorders, 49(6), $710-721$. https://doi.org/10.1111/1460-6984.12105

Satterthwaite, F. E. (1946). An approximate distribution of estimates of variance components. Biometrics bulletin, 2(6), 110-114. https://doi.org/10.2307/3002019

Skodda, S., \& Schlegel, U. (2008). Speech rate and rhythm in Parkinson's disease. Movement Disorders, 23(7), 985-992. https://doi.org/10.1002/mds.21996

Snedeker, J., \& Trueswell, J. (2003). Using prosody to avoid ambiguity: Effects of speaker awareness and referential context. Journal of Memory and Language, 48(1), 103-130. https://doi.org/10.1016/S0749$\underline{596 \times(02) 00519-3}$ 
Yorkston, K. M., Strand, E. A., \& Kennedy, M. R. T. (1996). Comprehensibility of Dysarthric Speech: Implications for Assessment and Treatment Planning. American Journal of Speech-Language Pathology, 5(1), 55-66. https://doi.org/10.1044/1058-0360.0501.55 